\title{
IDOSOS FRAGILIZADOS COM SINTOMAS DEPRESSIVOS ATENDIDOS NA ATENÇÃO PRIMÁRIA À SAÚDE
}

Aline de Sousa Falcão; Universidade Federal do Paraná (UFPR); E-mail: alinesousafalcão@hotmail.com;

Conceição da Silva Brito; UFPR; E-mail: enfbryto@gmail.com; Márcia Marrocos Aristides Barbiero; UFPR; E-mail: marciamarrocos@gmail.com;

Patrícia Rosa Gonçalves Leta; UFPR; E-mail: patriciarg|@hotmail.com;

Reuber Lima de Sousa; UFPR; E-mail: reuber_souza@hotmail.com; Karina Silveira de Almeida Hammerschmidt; UFPR; E-mail: ksalmeidah@ufpr.br; Susanne Elero Betiolli; Universidade Federal do Paraná; susanne.elero@yahoo.com.br; Maria Helena Lenardt; UFPR; E-mail: curitiba.helena@gmail.com

\section{RESUMO}

Introdução: a fragilidade física é uma condição multifatorial que possue potencial para afetar alguns aspectos da saúde dos idosos. Entre eles o de abalar a saúde mental, com o surgimento de sintomas depressivos. Objetivo: avaliar a fragilidade física de idosos com sintomas depressivos em acompanhamento na atenção primária à saúde. Métodos: trata-se de estudo quantitativo de corte transversal, desenvolvido em uma Unidade Básica de Saúde de Curitiba-Paraná (Brasil), com amostra composta por 389 idosos ( $\geqq 60$ anos). Realizou-se o prévio rastreio cognitivo, e coletaram-se os dados mediante questionário sociodemográfico, escala do Center for Epidemiologic Studies Depression (CES-D), e fenótipo da fragilidade física. Para as análises foram empregadas estatísticas descritivas. O projeto foi aprovado pelo Comitê de Ética em Pesquisa sob o número 2918847. Resultados: dos 389 idosos, 103 (26,4\%) possuíam sintomas depressivos, dos quais 75 (72,8\%) eram do sexo feminino, de 60 a 69 anos $(n=47 ; 45,6 \%)$, cor da pele autodeclarada branca $(n=74 ; 71,8 \%)$, casados $(\mathrm{n}=39 ; 37,8 \%)$ e aposentados $(\mathrm{n}=59 ; 57,2 \%)$. Do total de idosos com sintomas depressivos, $19(18,4 \%)$ eram frágeis, $63(61,2 \%)$ pré-frágeis e 21 (20,4\%) não frágeis. Conclusão: os resultados evidenciaram que entre os idosos com sintomas depressivos, houve predomínio do sexo feminino, aposentados e em condição de pré-fragilidade, no entanto, é considerável o quantitativo de idosos frágeis e com sintomas depressivos. Os dados sinalizam a necessidade de intervenções voltadas à gestão da fragilidade e aos idosos com sintomas depressivos da atenção primária à saúde.

Palavras-chave: Idoso Fragilizado; Depressão; Saúde do Idoso.

Agradecimentos: Os pesquisadores foram assegurados com o apoio financeiro da Coordenação de Aperfeiçoamento de Pessoal de Nível Superior - Brasil (CAPES) com concessão de bolsas de estudo do curso de pós-graduação (Mestrado). 